\title{
How is cell proprioception related to cell growth and differentiation? Strong scientific evidence for future clinical activities
}

\author{
David Rodriguez-Sanz ${ }^{1}$ \\ (iD) Marta Elena Losa-Iglesias² \\ Ricardo Becerro de Bengoa-Vallejo ${ }^{3}$ \\ (iD) Patricia Palomo-Lopez ${ }^{4}$ \\ (iD) Cesar Calvo-Lobo \\ (iD) Daniel Lopez-Lopez ${ }^{6}$
}

\begin{abstract}
1. Universidad Europea de Madrid. Faculty of Sport Sciences. Villaviciosa de Odón, Madrid, Spain 2. Universidad Rey Juan Carlos, Faculty of Health Sciences, Alcorcón, Madrid, Spain 3. Universidad Complutense de Madrid, School of Nursing, Physiotherapy, and Podiatry, Madrid, Madrid, Spain 4. Universidad de Extremadura, University of Center of Plasencia,Plasencia, Caceres, Spain 5. Universidad de León, Nursing and Physical Therapy Department, Faculty of Health Sciences León, León, Province of León, Spain 6. Research, Health and Podiatry Unit. Department of Health Sciences. Faculty of Nursing and Podiatry. Universidade da Coruña, Ferrol, Spain
\end{abstract}

\section{INTRODUCTION}

Why do cells care about physical stimuli?

Tissues are continuously subjected to the effects of different physical stimuli, with profound importance at clinical activities, and the interactions of these stimuli might affect cell development. We can observe different examples in the physical effects on cell growth, the results of gravitational force on the mineral status of bones, and the deficits in the mineral deposition in bones when the effects of gravity are absent. For example, astronauts can get osteoporosis after long periods in space, and the direct application of UVA improves skin healing in patients with psoriasis.

The spatial and temporal responses to physical phenomena are being investigated in detail to obtain in-depth knowledge of these responses and increase our understanding of the cellular nature and use of these physical elements to increase physiological responses, prevent pathological effects on the cell components and improve clinical activities in nursing, podiatry, physical therapy, and medicine among others ${ }^{1}$.

How do cells respond to physical stimuli?

At the basic level, the cell occupies a known and limited volume and might receive physical stimuli anywhere in it. Knowledge of biology, chemistry, and physics indicates that physical stimuli can assist in the growth, differentiation, and proliferation of cells and aid our understanding of the mechanisms by which these cells die.

This idea can serve as a strong starting point but must then be transferred to the next level of organi-

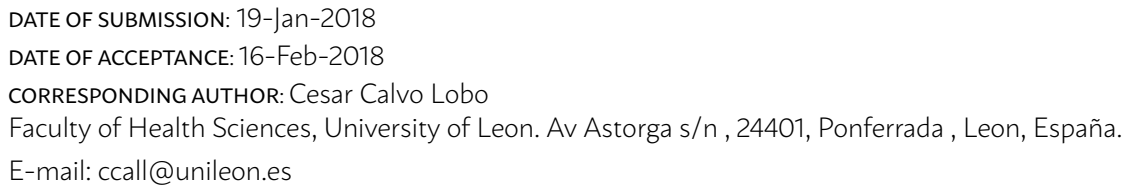


zation, which will promote the maintenance of tissue balance via physical stimuli to increase the health of tissues and organs and serve as a reliable tool for preventing pathology ${ }^{2}$. This concept will promote an exciting perspective for clinical professionals about these factors.

Similarly, when patients suffer an injury to an active tissue, such as a muscle-tendon unit or an articulation (e.g., an ankle sprain), one of the most important and useful techniques for promoting healing is known as perturbation training or proprioception. Proprioception is the process by which the body responds to incoming information about external forces by utilizing receptors and integrators to promote healing. These stimuli indicate that mechanoreceptors sense, integrate, and promote healing, and different responses are present in the muscle-tendon unit. We can translate this idea to the cellular level to observe how a cell responds via the strong actions of integrin to transfer physical stimuli and act as a local integrator that can generate different responses that develop into real cell proprioception that promotes healing and homeostasis at the cellular level. We propose that this complex mechanism should be defined as cell proprioception.

Moreover, the study of the relationship between the complex extracellular cell matrix (ECM) and stimuli is essential. An integrin-mediated mechanism can modify many cellular activities, such as cell-cell and cell-matrix interactions. There are other mechanosensors known to be important, such as GJS, cilium, and hemichannels.

Regarding integrins, these crucial elements can transfer mechanical stimuli from the matrix to the cytoskeleton through the formation of adhesion complexes. These complexes can redirect signal transductions (for example, via kinases) that influence the cytoskeleton and promote matrix remodelling ${ }^{4}$. This clinical approach is very interesting to handle clinical activities in all aggressive chemical and pharmacological cancer treatment approaches.

When we observe the action of the cytoskeleton, we can affirm that the behavior of the cytoskeleton is a crucial integrator ${ }^{5}$. This integration is mediated by the cytoskeleton tensegrity, which is led by two principal ideas. The first is structural self-remodeling. The second is the equilibrium between tensile and compressive forces (internal and external) that leads to the dynamic equilibrium that is so important for molecular changes ${ }^{4-6}$. The modification of passive cells and their neighbors by external stimuli alters cell-cell adhesion complexes ${ }^{7}$. Through such complexes, cells are joined to each other and the matrix to generate a dynamic system that is modified by stimuli at the cell level. We can understand the matrix as a controller of dynamic cell functions and behaviors and not only as the place where cells can be found.

Cells also use the physical characteristics of the ECM microenvironment to develop a traction-activated system that can influence both the cell and the $\mathrm{ECM}^{8}$. Therefore, we can appreciate the dynamic mechanical equilibrium between traction forces on the cell and the resistance points in the ECM, which generates a reciprocal isometric system of force ${ }^{9}$.

Many studies have provided strong evidence about how the interactions and modifications of this dynamic equilibrium can promote tissue regeneration. First, ECM elasticity can lead pluripotential cells to different evolutions within the same basal conditions, and in such situations, the only differences are related to the biophysical characteristics of the ECM and its geometric development ${ }^{10,11}$. Homeostasis can be properly handled by clinicians to promote a better development of this cell interactions.

The difference in stiffness across different ECMs can be associated with the loss of filaments or actin microtubules ${ }^{12}$. This biophysical model suggests that the cytoskeleton is an essential target of stimulation factors. Therefore, influences on the cytoskeleton can affect and modify the geometry of cell distributions via influences on cell development. Recent studies have shown that modified cell geometries can alter cell signaling and function ${ }^{13}$ and that modification to the cytoskeleton force can modify cell proliferation $^{14}$. Therefore, distortions of cell shapes can lead to stem cell evolution: Human MSCs are differently regulated if they promote or restrict ${ }^{15}$. It is important to remember that integrin acts as an essential mechanosensor that is helped by other known mechanosensors, such as CJS or hemichannels ${ }^{16}$.

Mechanotherapy that guides mechanotransduction signaling developments can be primarily targeted to control the following four processes ${ }^{17}$ : (a) the mechano-coupling phase, the external physical stimuli is transformed into a mechanical signal; (b) biochemical coupling, in which the local signal is transduced into a biochemical signal; (c) signal transmission in which the signal is then passed from the signal biochemistry receptor cells to the effector 
cells; and (d) the response of the effector cells. A recent study has reported on the control of the union of the external forces, and biochemical signaling networks spatial-mechanical regulation controls cell; the simple mechanical restriction of motion surface molecules can alter cell behavior.

\section{Distinguishing the mechanisms of}

physical stimuli, gravitational force, and

electromagnetic force

Multiple physical stimuli can work in a combined or straightforward manner on the cell interactions. It is important to know both the most profound nature of the cell and its characteristics and the most profound nature of the stimuli with which the cell interacts. By analyzing the different forces that can influence a cell, we can observe, at the most fundamental level, what appears to be the so-called fundamental forces those forces are the forces of the universe that cannot be explained like other more basic forces. There are four of these fundamental forces: gravitational, electromagnetic, strong nuclear and weak nuclear. Clinicians can frequently use gravitational and electromagnetic forces to improve healing levels in patients The gravitational force is the attraction that is exerted on the subject matter and affects all bodies. Gravity is feeble and is a one-way force but is of infinite extent. At the cellular level, we can understand the force of gravity as existing between all cells; we could understand the gravitational force as externally inducing the active system of ECM-cell or cells. The electromagnetic force affects electrically charged bodies, and this force is involved in the physical and chemical transformations of the atoms and molecules. Molecules are located at the cell level before the organizational level. This force is much stronger than the gravitational force and has two meanings (positive and negative), with infinite range. Moreover, we can externally induce an electromagnetic field to influence the cell and the ECM-cell system. These physical effects are deeply known by clinicians, podiatry doctors, or physiotherapist.

The nuclear force or strong interaction is the component that holds atomic nuclei and acts interchangeably between any two nucleons, protons or neutrons. The scope of this force is on the order of nuclear dimensions, but it is stronger than the electromagnetic force.

The nuclear force or weak interaction is responsible for the beta decay of neutrons; neutri- nos are only sensitive to this type of interaction. The intensity of this force is lower than that of the electromagnetic force, and its range is even smaller than the strong nuclear interaction. Everything that happens in the universe and therefore in the cell is due to the action of one or more of these forces, whether alone or combined, and these forces differ from each other in that each involves the exchange of different particle types. These particle exchanges are denominated or intermediary. All exchanged particles are bosons, while the interaction source particles are fermions. Currently, scientists are attempting to prove that all of these apparently different fundamental forces are manifestations of a single mode of interaction in different circumstances. There are various theories. The term "unified field theory" encompasses new theories in which two or more of the four fundamental forces appear as if they are basically identical. The theory of everything is another unified field theory that aims to provide a unified description of the four fundamental forces. Currently, the best candidate to become a theory of everything is superstring theory.

Physical stimuli might promote important cell effects; therefore, we can transfer it to clinical fields to influence and change the associated biological processes. Physical stimuli are essential for obtaining tissue homeostasis and common clinical treatments. It can lead to and help the cells to check and modify their behavior for a new relationship with new and altered environmental conditions. Consequently, a strong candidate for inducing optimal regeneration, promoting homeostasis and promoting the cell repair process is the contribution of physical stimuli in a controlled and correct manner. The most essential elementary forces studied enable us to externally induce cell and ECM-cell complexes regarding gravitational and electromagnetic forces, which we will describe below. These physical effects are deeply known by clinicians, podiatry doctors or physiotherapist.

\section{GRAVITATIONAL FORCE}

The influence of gravity as a direct mechanical vector in leading cell processes has a crucial historical antecedent. Galileo studied how bone modification is associated with vector components and their influences. The papers of Rubin and Ingber are strongly knowledgeable ${ }^{18-20}$. Cells are subject to the effects of several physical stimuli that are caused by among 
other things, gait, posture, and respiration, and generate different possibilities. It has been shown that changes in standard components can modify cell complexes in important manners. Cells can receive stimuli that are moved through membrane receptors and are transferred to the cell cytoskeleton to the ECM and other cells. These factors modify the ECM mechanistic behavior and cell shape ${ }^{20}$. The extracellular matrix is an essential elastic structure that is continuously renewed, particularly concerning the cell environment. The ECM is associated with cells that offer relevant information and physical stimuli factor into the structural and external forces ${ }^{21}$. Stimulus factors change ECM homeostasis; e.g., physical stimuli help to control the production of ECM components through, for example, the activation of intracellular signaling pathways ${ }^{22}$.

The in vitro test of different cells with different physical stimuli, such as mechanical stretch, simulate the mechanical action of heart cells, which exhibit morphological activations that are similar to those that occur during heart growth ${ }^{15,23}$. It is important to remember that internal and external stimuli can influence cell shape and that recent trials have demonstrated that cell shape can lead to apoptosis and different cell actions such as gene expression and protein synthesis ${ }^{24}$. Simulations of the effects of gravity, pairs of real gravity and modeling of gravity with random positioning machines (RPMs) could be difficult for the cellular organization and ECM creation in immature cartilage ${ }^{25}$. Different studies have shown how gravitational effects, after $72 \mathrm{~h}$ of microgravity exposure, result in cytoskeletal reorganization, and the important osteoclastic markers of nuclear factor kappa-B and receptor activator of nuclear factor kappa-B ligand are solidly augmented to affect the ability of bone reabsorption ${ }^{26}$. These studies, in concordance with other authors ${ }^{27,28}$, promote the notion that mechanical or gravitational stimuli influence osteoblastic differentiation.

In contrast, gravitational effects and decreases in mechanical stimuli cause osteoclastogenesis and bone tissue resorption or modifications of laminin and fibronectin production by fibroblasts ${ }^{29}$. These modifications of fibronectin might be the cause of altered wound-healing responses ${ }^{30,31}$

In contrast, following controlled hypergravity exposure (10xg), we did not find significant changes in cell metabolism or cell anatomy. Findings have been published that state that hypergravity effects main- tain endothelial cell survival and the actions of the activation of adaptive characters ${ }^{32}$.

For example, in 1997, Chen et al. ${ }^{33}$ developed a trial using mechanical stimuli and obtained significant improvements and differentiation of human endothelial cells. Later, Chiquet et al. ${ }^{34}$ used mechanical stress mediated by stretching with 5 to $15 \%$ elongation and a frequency of 0,3 a $1 \mathrm{~Hz}$ and observed an increase in extracellular matrix components (mainly tenascin C). In 2006, Park et al. ${ }^{35}$ also used stretching as mechanical stimuli with a frequency of 0,5 $\mathrm{Hz}$ and $8 \%$ intensity in fibroblasts and found that obtaining a cell growth and proliferation as well as collagen. In 2007, Vatsa et al. ${ }^{36}$ developed mechanical stress microneedles into osteocytes by increasing the regulation of NO-stimulated cell growth. In 2008, Monici et al. ${ }^{37}$ developed a stress stimulus with hypergravity and generated $10 \mathrm{~min}$ cycles of $10 \mathrm{~g}$ with 10 min of recovery at $1 \mathrm{~g}$ in human mesenchymal cells and obtained an overexpression of genes related to osteoblastogenesis. In 2011, Chan et al. ${ }^{38}$ conducted a study on the compressive mechanical effects ranging from 5 to $20 \%$ with a frequency of 0.15 at 1 $\mathrm{Hz}$ and a duration of 1-12 h/day at a hydrostatic pressure of 0,1 to $10 \mathrm{MPa}$ and held on intervertebral discs and stem cells. These authors found that phenotypic therein affects discogenic differentiation. Grad et al. ${ }^{39}$ developed a mechanical stimulus with uniaxial and multiaxial loadings of 7 to $10 \mathrm{MPa}$ of stress in chondrogenic cells and observed that a regulation of the chondrogenic patterns. In 2011, Maul et al. ${ }^{40}$ performed mesenchymal stem cell stimulation via mechanical stretch stimuli at $5 \%$ with a frequency of 1 $\mathrm{Hz}$ and increased the expression of the same.

Electromagnetic force

In the ancient times of the first century $\mathrm{AD}$, we found proof of the use of electric fish to treat a headache. In the $15^{\text {th }}$ century AD, Paracelsus studied the medical use of electricity, and later Digby described the use of the magnetic field in wound treatment. Then, Galvani led the ideas for actual research lines related to physiological electromagnetic fields and their effects.

In the last four decades, in-depth knowledge has been acquired related to the understanding of bioelectricity effects; changes in electrical membrane potentials of the living have been shown to result in modifications of potential gradients that have been associated with morphogenetic changes ${ }^{41}$. Thus, many physiological events have been localized to the 
critical tool-signals for processes in development ${ }^{42}$.

An in-depth and consistent analysis of the electrical and magnetic implications of cell biology is shown in a paper written by Funk et al. ${ }^{43}$. This paper demonstrated the association between physical stimuli and cell biology integration processes. EMFs can influence the polarization of bound charges, the orientation of dipoles, the diffusion of charges, ion-channel and receptor modifications, conformational modifications of voltage-sensitive enzymes and essential modifications of membrane phospholipids with different activation kinetics of the ion channels ${ }^{43}$.

The modifications of EMFs created by living tissues are generated by different physiological activities; for example, muscle contraction is known for the existence of vibrations of muscles that promote direct mechanical strain and currents via both posture $(5-30 \mathrm{~Hz})$ and gait $(<10 \mathrm{~Hz})$ and have been studied ${ }^{44}$. Muscle contractions generate bone tissue EMFs, which are essential for maintaining bone homeostasis. Bone cells are particularly sensitive to low frequencies (in the range of 15 to $30 \mathrm{~Hz}$ ). In this range, electromagnetic fields of $0.01 \mathrm{mV} / \mathrm{cm}$ aid bone remodeling ${ }^{18,19}$. It has been found that the EM activation produced by mechanical loading $(1 \mathrm{~Hz}$ during walking) in bone promotes the range of $0.1-1.0 \mathrm{~mA} /$ $\mathrm{cm} 2{ }^{45}$. In general terms, physiologically endogenous EMFs are characterized by low frequencies (ELF) from 0 to $300 \mathrm{~Hz}$ and low intensity.

EMFs field interventions are frequently used in the treatment of musculoskeletal disorders, and many studies have indicated that the most effective applications are associated with pulsed EMFs in the range of 1 to $100 \mathrm{~Hz}$; this range promotes $\mathrm{EF}$ on the order of $\mu \mathrm{V} / \mathrm{cm}^{46}$. Thus, physiological benefits may be induced by low-frequencies and -amplitude EMFs (range 8-60 Hz) ${ }^{44}$. It has been shown that pulsed EMFs can stimulate osteoblastic differentiation and proliferation or inhibit osteoclastogenesis, and both of these bone processes are important to bone homeostasis ${ }^{47,48}$.

Recent trials have shown the possibility of applying EMFs to promote ligament healing processes and repair and aiding ligament homeostasis after a pulsed EMF stimulates fibroblasts to improve migration speed and enhance collagen I expression. Ligaments and tendons are similar in their healing processes, and tendon can be favored by pulsed EMFs.

Conversely, static EMFs slow the wound healing process. In contrast, pulsed EMFs can improve this process $^{49}$. We affirm that EMFs modulate cell proliferation, and both of the EMF components of intensity and frequency are fundamental in the final effect. Regarding magnetism, Kwee et al..$^{50}$ showed an increase in human fibroblast proliferation following exposure to $0.08 \mathrm{mT}$. Kula et al. ${ }^{51}$ demonstrated an inhibition of cell growth in fibroblasts exposed to $20 \mathrm{mT}$. Different trials that have been performed by exposing different cultures have shown different effects (i.e., increases, decreases or no effect) that are intensity-dependent ${ }^{52}$.

Regarding frequency, many authors have shown significant increases in the proliferation of different cell types at a rate of $50 \mathrm{~Hz}^{53}$. Several trials have designated the interaction between EMFs and calcium fluxes because calcium is an essential regulator of many cellular processes and leads to the activation of the cyclic AMP, which is a crucial trigger molecule in intracellular metabolic processes. It has been shown that EMFs can modify calcium concentrations in different ways that depend on cell type and field intensity ${ }^{54}$.

The effects of EMFs on cell differentiation have also been intensely studied. A progressive inhibition of enzyme activity and differentiation in osteoblasts that follows exposure to $30 \mathrm{~Hz}$ EMF was described by McLeod and Collazo ${ }^{55}$. In cultures exposed to EMFs during chondrogenic differentiation, it has been observed that collagen II and glycosaminoglycan increase ${ }^{48}$. Therefore, EMFs might be a way to promote and maintain chondrogenesis and to illustrate new steps in regenerative treatment for cartilage.

Preliminary experiments have shown the effect of pulsed EMFs on the role of fibroblasts in wound healing and aligned with other authors ${ }^{57}$. EMFs can influence the acceleration or slowing of the migration of fibroblasts, depending on the properties of the applied field. The possibility of modulating fibroblast migration during wound healing could be very interesting. Indeed, this possibility might be useful for enhancing the migration of fibroblasts to promote wound healing in chronic ulcers and general cases in which healing is slow. Inhibiting migration would be beneficial for preventing the formation of dysfunctional scars.

In 1993, McLeod and Rubin ${ }^{45}$ assessed the effects of pulses of 15 to $30 \mathrm{~Hz}$ with a characteristic of 0.01 $\mathrm{mV} / \mathrm{cm}$ on bone cells with the goal of improving remodeling activity. In 1995, Kwee \& Raskmark ${ }^{50}$ con- 
ducted a study of human fibroblast proliferation in an electromagnetic field of $50 \mathrm{~Hz}$ with an intensity of 25-180 microtesla and found an increase in cell proliferation. In 2002, Harting et al. ${ }^{47}$ conducted a study of an electric field of $100 \mathrm{v}$ to $16 \mathrm{~Hz}$ in osteoblastic cells and found proliferation, increased expression of alkaline phosphatase and an improvement in the production of extracellular matrix synthesis. In 2004, Chang et al. ${ }^{48}$ appreciated the effects of an electromagnetic field with a frequency of $15 \mathrm{~Hz}$ and an intensity of 0.1 $\mathrm{mT}$ on osteoblastic populations and found an increase in osteoblast proliferation. In 2010, Mayer-Wagner et al. ${ }^{56}$ studied the effect of electromagnetic fields with frequencies of $15 \mathrm{~Hz}$ and intensities of $5 \mathrm{MT}$ on mesenchymal cells and found an increased production of type II collagen and glycosaminoglycans during chondrogenic differentiation.

\section{CONCLUDING THOUGHTS}

Interaction of cell-physical stimuli

The cell continuously receives physical stimuli, and these stimuli can directly influence the future evolution of the cell. Different characteristics of the physical quantities are of great interest in defining and meeting their influence on cells.

1. The theory of the right stimulus. The first principle of cell proprioception.

a) The quality of the nature of the physical stimuli, either scalar or vector, must be appropriate for each cell type and each time in different cell cycles in which it is to generate a favorable effect on the development and/or cell differentiation thereof if no adequate quality can have a negative impact on their differentiation and/or development or have a neutral effect.

b) The amount of the physical stimuli, either sca- lar or vector, must be appropriate for each cell type and for each different cell cycle in which it is to generate a beneficial effect on the development and/or differentiation time if no adequate quality can have a negative impact on their differentiation and/or development or have a neutral effect.

c) The temporal components of physical stimuli, such as frequency and duration that interact with the cell must be appropriate for each cell type and for each different cell cycle in which it is to generate a beneficial effect on the development and/or differentiation time. If the temporal component is not adequate, it might have a negative effect on their differentiation and/or development or have a neutral effect.

d ) The spatial components of the physical stimuli that interact with the cell and the cellular environment must be appropriate for each cell type and for each different cell cycle in which it is to generate a beneficial effect on the development and/or differentiation, if the spatial component is not adequate may have a negative effect on their differentiation and/or development or have a neutral effect,

2. The theory of right equivalent stimulus. The second principle of cell proprioception.

a. Two physical stimuli generating an effect equivalent to a third stimulus effect are equivalent among themselves.

b. If the sum of the effects of physical stimuli that interact are equal to the sum of other different physical stimuli that interact with one another, the resulting stimulus is equivalent to both interactions.

c. If two physical stimuli of different natures produce the same effect; they are equivalent.

d. The sum of the effects of different physical stimuli is different from the sum of the separate parts due to the interference of the same.

\section{REFERENCES}

1. Monici M, Cialdai F. The role of physical factors in cell differentiation, tissue repair and regeneration. In: Jamie D, ed. From basic biology to clinical application. Rijeka: IntechOpen;2012. p.13-34.

2. Huang C, Holfeld I, Schaden W, Orgill D, Ogawa R. Mechanotherapy: revisiting physical therapy and recruiting mechanobiology for a new era in Medicine. Trends Mol Med. 2013;19(9):555-64.

3. Ingber DE. Mechanical signaling and the cellular response to extracellular matrix in angiogenesis and cardiovascular physiology. Circ Res. 2002;91(10):877-87.
4. Ingber DE. Tissue adaptation to mechanical forces in healthy, injured and aging tissues. Scand J Med Sci Sports. 2002;15(4):199-201.

5. Ingber DE. The mechanochemical basis of cell and tissue regulation. Mech Chem Byosyst. 2004;1(1):53-68.

6. Ingber DE. The architecture of life. Sci Am. 1998;278(1):48-57.

7. Ingber DE. Tensegrity II. How structural networks influence cellular information processing networks. J Cell Sci. 2003;116(Pt 8):1397-408.

8. Wang JH, Lin JS. Cell traction force and measurement methods. Biomech Model Mechanobiol. 2007;6(6):361-71. 
9. Paszek MJ, Weaver VM. The tension mounts: mechanics meets morphogenesis and malignancy. J Mammary Gland Biol Neoplasia. 2004;9(4):325-42.

10. Engler AJ, Sen S, Sweeney HL, Discher DE. Matrix elasticity directs stem cell lineage specification. Cell. 2006;126(4):677-89.

11. Pathak A, Kumar S. Independent regulation of tumor cell migration by matrix stiffness and confinement. Proc Natl Acad Sci U S A 2009;109(26):10334-9

12. Lekka M, Laidler P, Gil D, Lekki J, Stachura Z, Hrynkiewicz AZ. Elasticity of normal and cancerous human bladder cells migration by scanning force microscopy. Eur Byophys J. 1999;28(4):312-6.

13. Alford PW, Nesmith AP, Seywerd IN, Grosberg A, Parker KK. Vascular smooth muscle contractility depends on cell shape. Integr Biol (Camb) 2011;3(11):1063-70

14. Huang S, Chen CS, Ingber DE. Control of cyclin D1, p27(Kip1), and cell cycle progression in human capillary endothelial cells by cell shape and cytoskeletal tension. Mol Biol Cell. 1998;9(11):3179-93.

15. McBeath R, Pirone DM, Nelson CM, Bhadriraju K, Chen CS. Cell shape, cytoskeletal tension, and RhoA regulate stem cell lineage commitment. Dev Cell. 2004;6(4):483-95.

16. Donahue HJ. Gap junctions and biophysical regulation of bone cell differentiation. Bone. 2000;26(5):417-22.

17. Turner $\mathrm{CH}$, Pavalko FM, Mechanotransduction and functional response of the skeleton to physical stress: the mechanisms and mechanics of bone adaptation. J Orthop Sci. 1998;3(6):346-55.

18. Rubin CT, Lanyon LE. Regulation of bone formation by applied dynamic loads. J Bone Joint Surg Am. 1984;66(3):397-402.

19. Rubin C. Skeletal strain and the functional significance of bone architecture. Calcif. Tissue Int. 1984;36(suppl 1):11-8.

20. Ingber DE, Wang N, Stamenovic D. Tensegrity, cellular biophysics, and the mechanics of living systems. Rep Prog Phys. 2014;77(4):046603.

21. Carson DD. Extracellular matrix: forum introduction. Reprod Biol Endocrinol. 2004;2:1.

22. Chiquet M, Gelman L, Lutz R, Maier S. From mechanotransduction to extracellular matrix gene expression in fibroblasts. Biochim Biophys Acta. 2009;1793(5):911-20

23. Vandenburg HH, Solerssi R, Shansky I, Adams JW, Henderson SA. Mechanical stimulation of organogenic cardiomyocyte growth in vitro. Am Physiol. 1996;270(5 Pt 1):C1284-92.

24. Thomas $\mathrm{CH}$, Collier JH, Sfeir CS, Healy KE. Engineering gene expression and proteins synthesis by modulation of nuclear shape. Proc Natl Acad Sci U S A. 2002:99(4):1972-7.

25. Stamenkovic I, Yu Q. Merlin, a 'magic' linker between extracellular cues and intracellular signaling pathways that regulate cell motility, proliferation and survival. Curr Protein Pept Sci. 2010;11(6):471-84.

26. Monici M, Fusi F, Paglierani M, Marziliano N, Cogoli A, Pratesi R, et al. Modeled gravitational unloading triggers differentiation and apoptosis in preosteoclastic cells. J Cell Biochem. 2006;98(1):65-80.

27. Kaneuji T, Ariyoshi W, Okinaga T, Toshinaga A, Takahashi T, Nishihara T. Mechanisms involved in regulation of osteoclastic differentiation by mechanical stress-loaded osteoblasts. Biochem Biophys Res Commun. 2011;408(1):103-9.

28. Wan L, Zhang X, Guo Y, Chen X, Li R, Liu L, et al. Involvement of BMPs/ Smad signaling pathway in mechanical response in osteoblasts. Cell Physiol Biochem. 2010;26(6):1093-102.

29. Monici M, Cialdai F, Romano G, Fusi F, Egli M, Pezzatini S, et al. An in vitro study on tissue repair: impact of unloading on cells involved in the remodelling phase. Microgravity Sci Technol. 2011;23(4):391-401.

30. Midura RJ, Su X, Androjna C. A simulated weightlessness state diminishes cortical bone healing responses. I Musculoskelet Neuronal Interact. 2006;6(4):327-8

31. Delp MD. Unraveling the complex web of impaired wound healing with mechanical unloading and physical deconditioning. J Appl Physiol. 2008;104(5):1262-3

32. Morbidelli L, Marziliano N, Basile V, Pezzatini S, Romano G, Conti A, et al. Effect of hypergravity on endothelial cell function and gene expression. Microgravity Sci Technol. 2009;21(1-2):135-40.

33. Chen CS, Mrksich M, Huang S, Whitesides GM, Ingber DE. Geometric control of cell life and death. Science. 1997:276(5317):1425-8.
34. Chiquet M, Renedo AS, Huber F, Flück M. How do fibroblast translate mechanical signals into changes in extracellular matrix production? Matrix Biol. 2003;22(1):73-80

35. Park SA, Kim IA, Lee YJ, Shin JW, Kim CR, Kim JK, et al. Biological responses of ligament fibroblasts and gene expression profiling on micropatterned silicone substrates subjected to mechanical stimuli. J Biosci Bioeng. 2006;102(5):402-12.

36. Vatsa A, Smit TH, Klein-Nulend J. Extracellular NO signaling from a mechanically stimulated osteocyte. | Biomech. 2007;40(Suppl 1):S89-95.

37. Monici M, Romano G, Cialdai F, Fusi F, Marziliano N, Benvenuti S, et al. Gravitational/mechanical factors affect gene expression profile and phenotypic specification of human mesenchymal stem cells. J Gravit Physiol. 2008;15(1):191-2

38. Chan SC, Ferguson S|, Gantenbein-Ritter B. The effects of dynamic loading on the intervertebral disc. Eur Spine J. 2011;20(11):1796-812.

39. Grad S, Eglin D, Alini M, Stoddart MJ. Physical stimulation of chondrogenic cells in vitro: a review. Clin Orthop Relat Res. 2011;469(10):2764-72.

40. Maul TM, Chew DW, Nieponice A, Vorp DA. Mechanical stimuli differentially control stem cell behavior: morphology, proliferation, and differentiation. Biomech Model Mechanobiol. 2011;10(6):939-53.

41. McCaig CD, Rajnicek AM, Song B, Zhao M. Controlling cell behavior electrically: current views and future potential. Physiol Rev. 2005;85(3):943-78.

42. Levin M. Large-scale biophysics: ion flows and regeneration. Trends Cell Biol. 2007;17(6):261-70.

43. Funk RH, Monsees T, Ozkucur N. Electromagnetic effects: from cell biology to medicine. Prog Histochem Cytochem. 2009;43(4):177-264.

44. Antonsson EK, Mann RW. The frequency content of gait. I Biomech. 2008;18(1):39-47.

45. McLeod KJ, Rubin CT. Observations from mechanically and electrically induced bone remodeling. In: Blank M, ed. Electricity and magnetism in biology and medicine. San Francisco:San Francisco Press; 1993. p.70-98.

46. Pilla AA. Low-intensity electromagnetic and mechanical modulation of bone growth and repair: are they equivalent? | Orthop Sci. 2002;7(3):420-8.

47. Hartig M, Joos U, Wiesmann HP. Capacitively coupled electric fields accelerate proliferation of osteoblast-like primary cells and increase bone extracellular matrix formation in vitro. Eur Biophys |. 2000;29(7):499-506.

48. Chang WH, Chen LT, Sun JS, Lin FH. Effect of pulse-burst electromagnetic field stimulation on osteoblast cell activities. Bioelectromagnetics. 2004:25(6):457-65.

49. Chao $\mathrm{PH}$, Lu HH, Hung CT, Nicoll SB, Bulinski IC. Effects of applied DC electric field on ligament fibroblast migration and wound healing. Connect Tissue Res. 2007;48(4):188-97.

50. Kwee S, Raskmark P. Changes in cell proliferation due to environmenta non-ionizing radiation. 1. ELF electromagnetic fields. Bioelectrochem \& Bioenerg. 1995;36(2):109-14

51. Kula B, Drozdz M. A study of magnetic field effects on fibroblast cultures. Part 1. The evaluation of effects of static and extremely low frequency (ELF) magnetic fields on vital functions of fibroblasts. Bioelectrochem \& Bioenerg. 1996;39(1):21-6

52. Scarfi MR, Lioi MB, Zeni O, Della Noce M, Franceschi C, Bersani F. Micronucleus frequency and cell proliferation in human lymphocytes exposed to $50 \mathrm{~Hz}$ sinusoidal magnetic fields. Health Phys. 1999;76(3):244-50.

53. Cossarizza A, Capri M, Salvioli S, Monti D, Franceschi C, Bersani F, et al. Electromagnetic fields affects cell proliferation and cytokine production in human cells. In: Blank M, ed. Electricity and magnetism in biology and medicine. San Francisco: San Francisco Press; 1993. p.640-2.

54. Walleczek J, Liburdy RP. Nonthermal $60 \mathrm{~Hz}$ sinusoidal magnetic-field exposure enhances 45Ca2+ uptake in rat thymocytes: dependence on mitogen activation. FEBS Lett. 1990;271(1-2):157-60.

55. McLeod KJ, Collazo L. Suppression of a differentiation response in MC 3T3-E1 osteoblast-like cells by sustained, low-level, $30 \mathrm{~Hz}$ magnetic-field exposure. Radiat Res. 2000;153(5 Pt 2):706-14

56. Mayer-Wagner S, Passberger A, Sievers B, Aigner J, Summer B, Schiergens $T$, et al. Effects of low frequency electromagnetic fields on the chondrogenic differentiation of human mesenchymal stem cells. Bioelectromagnetics. 2010;32(4):283-90.

57. Sunkari VG, Aranovitch B, Portwood N, Nikoshkov A. Effects of a low-intensity electromagnetic field on fibroblast migration and proliferation. Electromagn Biol Med. 2011;30(2):80-5. 